\title{
A Case of MELAS Presenting as Anorexia Nervosa
}

\section{To the Editor:}

The case presented describes a 24-year-old female with mitochondrial myopathy, encephalopathy, lactic acidosis, and stroke (MELAS) who was diagnosed with anorexia nervosa (AN) and treated for the eating disorder for two years before her mitochondrial cytopathy was detected. Upon closer scrutiny of her symptoms, she did not have body image disturbance and thus did not meet criteria for AN despite a body mass index (BMI) of 12 and significant reduction of food intake. Additionally, neurological and gastrointestinal symptoms, for which an eating disorder could not account, were present, prompting a broadening of the differential leading to the correct diagnosis. Eating disorder symptoms have been documented to occur comorbidly with a mitochondrial cytopathy but not as a presenting syndrome in the absence of an eating disorder as defined by the Diagnostic and Statistical Manual of Mental Disorders, Fourth Edition, Text Revision (DSM-IV- TR).

\section{CASE REPORT}

MELAS is a mitochondrial cytopathy defined by myopathy, encephalopathy, lactic acidosis and strokelike episodes. ${ }^{1-3}$ Stroke like episodes do not always correspond to the main cerebral arterial territories because the mechanism of cellular injury may be due to mitochondrial dysfunction rather than vessel ischemia. ${ }^{4}$ Ragged red fibers, detectable on muscle biopsy, are the result of an accumulation of ultrastructurally abnormal mitochondria that results in decreased aerobic adenosine triphosphate (ATP) production and impairment of energy-requiring cellular functions. ${ }^{5}$ Such aberrant mitochondria are found in various tissues throughout the body. Pathology occurs most commonly, but not exclusively, in tissues with high demands for ATP, such as those of the cardiac and skeletal muscles, and the central nervous system (CNS). ${ }^{6}$

The most often identified mitochondrial transfer RNA gene point mutation is at position 3243 (Adenine
[A] to Guanine [G]) in the mitochondrial transfer RNA gene for leucine, occurring in $86 \%$ of MELAS cases. The clinical expression of MELAS is highly variable for two main reasons. First, heteroplasmy exists among mitochondria within the same tissue. Secondly, the genetic load of mutant genomes that produces clinically apparent disease varies between tissues and organ systems. ${ }^{6,7,8}$

This letter focuses on gastrointestinal manifestations, psychiatric manifestations, and the interplay between them that may appear consistent with an eating disorder. Gastrointestinal symptoms may include early satiety, recurrent vomiting, diarrhea, and pseudo-obstruction. ${ }^{8}$ Psychopathological features of MELAS have been described and include disturbed impulse control and aggression, depression, affective instability, suicidal behavior, schizophrenialike psychosis, and cognitive decline. ${ }^{9}$

We present the case of a 24-year-old, married female with undiagnosed MELAS who had been treated for AN 2 years prior to hospitalization. The patient had been treated in the two year period prior to hospitalization for $\mathrm{AN}$ as a psychiatric outpatient. She had maintained her body weight at approximately 70 pounds, which for her height of 60 inches correlated with a BMI of 13.7. Approximately 2 months prior to hospitalization, she suffered a right-sided frontoparietal stroke with sequelae of left sided weakness, bilateral hearing loss, and new onset simple partial seizures. Subsequently, she experienced further weight loss down to 64.25 pounds (BMI of 12).

Upon admission, the patient reported desire to gain weight and denied food restricting or purging. The patient did complain of early satiety. She reported a longstanding history of low weight. Her highest adult weight, in fact, was 78 pounds and occurred at the time of high school graduation. At this weight she felt more attractive and perceived her peers as "envious" of her appearance. She stated that at her current weight she felt too thin. She 
denied a fear of becoming fat or placing undue emphasis on her weight or shape in her self evaluation. She denied mood disturbance, anxiety, or symptoms of psychosis.

The patient was not aware of any family history of neurological or psychiatric illness. She endorsed no previous history of stroke, neurological conditions, testing or prenatal screening for genetic abnormalities, or history of developmental disorders in any first degree relatives. Review of systems was significant for constipation and seven pound weight loss since her stroke. A developmental history was positive for sexual abuse in childhood, as well as verbal abuse persisting into adulthood. The patient had been a perfectionistic student and high achiever with no legal or disciplinary difficulties. She participated in cheerleading during high school. Her mother reported reduction of food intake beginning in her teen years. The patient's mother also described frequent parental struggles over eating. The patient's mother further described a close but highly conflicted relationship between the patient and her sister, and fierce competitiveness between them regarding appearance and especially weight. The patient's 22-year-old sister was 64 inches tall and 80 pounds, with a BMI of 13.7 .

Initial mental status exam revealed an emaciated, waif-like Caucasian female. She appeared and dressed as if she were much younger than her stated age. Her behavior was cooperative but childlike, and she displayed significant psychomotor retardation. Marked speech latency and slurred speech were observed. Judgment and insight were not grossly impaired.

Physical exam revealed generalized muscle atrophy, minimal breast development, and poor dentition. The patient was tachycardic with normal rhythm and normal S1, S2. The neurologic examination revealed $3 / 5$ weakness in the left upper extremity in the setting of generalized $4 / 5$ weakness. She had grossly intact Cranial Nerves III, IV, and V with poor tracking bilaterally. Sensory exam was unremarkable. Deep tendon reflexes were absent bilaterally. Poor sharp/dull discrimination was evident on the left. Cerebellar functioning was normal. The patient had a small, shuffling, left deviated gait. Laboratory studies including complete blood count, chemistry panel, and albumin were normal. The electrocardiogram showed no acute changes.

No binge eating, purging, or inappropriate compensatory behaviors were noted during her hospitalization. She was noted to eat only a cup of food at mealtimes but reported this was due to a feeling of abdominal discomfort. Collateral information from her husband was consistent with the patient's denial of restrictive eating or purging behaviors.
Given her physical findings, including the history of stroke and seizure, consultation with neurology was sought. On hospital day 5 , simple partial motor seizures were observed. Transthoracic electrocardiogram was negative. Magnetic resonance imaging and angiogram (MRI/MRA) of the patient's carotids was normal. MRI/MRA brain scans revealed confluent edema in both parietal lobes and infarction in the posterior temporal lobes, bilaterally, in the absence of any cerebral or cerebellar atrophy. Electroencephalogram showed diffuse slowing with brief bursts of high amplitude slow waves and no epileptiform discharges. Endoscopy and colonscopy were normal. A serum lactate level was $5.0(R R=0.5,-2.2)$.

On hospital day 14, the patient developed severe nausea and vomiting with abdominal pain, fever, and leukocytosis. Abdominal and pelvic computed tomography revealed a pseudo obstruction, which responded to decompression. On hospital day 19, she was able to resume her normal diet and was participating in physical and occupational therapy. She was discharged to a rehabilitation facility. Genetic testing for MELAS was ordered but at the time of discharge was incomplete. Based on the provisional diagnosis of MELAS, treatment with Lcarnitine and $\mathrm{COQ} 10$ was initiated. ${ }^{10}$

$\mathrm{BMI}$ at discharge was still 12. Weight at discharge was $65 \mathrm{lbs}$. Genetic testing on mitochrondrial transfer RNA (tRNA) obtained from muscle biopsy later revealed that the patient had a point mutation of MELAS tRNA Leu A3243G.

\section{DISCUSSION}

MELAS and AN may appear clinically similar in several aspects, especially in terms of neurological and gastrointestinal manifestations. Both conditions may be associated with emaciation. Food restriction may be present in both conditions. In MELAS, the motivation for restricting food may be related to physical discomfort," whereas in AN food restriction is based on desire for thinness or fear of being fat. ${ }^{12}$ A disturbed body image and fear of being fat are not present in MELAS but are criteria for AN.

Structural alterations in both MELAS and AN typically comprise ventricular enlargement and atrophy, though in MELAS atrophy may tend to be more asymmetric and involve a number of structures that tend to remain unaffected in AN, including the cerebellum, the parahippocampal gyrus, and the thalamus. Neuropathological similarities between MELAS and AN comprise neuronal loss with gliosis, however MELAS patients typically also have focal cortical lesions characterized by necrosis in the cerebral cortex, amygdala, hippocampus, and cerebellum. Cerebellar cortical degeneration of granular cell type, microcyst formation, and capillary proliferation may also be observed in MELAS. ${ }^{13}$ 
Cognitive changes may be present in patients with MELAS as well as patients with AN. In AN, cognitive symptoms may antedate the onset of the eating disorder. Patients with AN may be disproportionately impaired on tasks requiring visuospatial functioning. This observation, however, is not universally supported; more recent evidence suggests global cognitive impairments in patients with AN. There is accumulating evidence that MELAS patients may also have cognitive dysfunction due to adverse effects of oxidative stress. Mitochondria are essential organelles for neuronal function because, differing from muscle, the limited glycolytic capacity of these cells makes them highly dependent on aerobic oxidative phosphorylation for their energetic needs. ${ }^{14}$

It is unlikely but possible that the patient had both MELAS and AN syndrome. We ruled out this possibility based on a review of the criteria for AN in the DSM-IV-TR." Criteria for AN include fear of gaining weight and becoming fat, disturbed body image, undue emphasis on shape or weight on self evaluation, and either restricting or binge eating/purging behaviors. In contrast, our patient stated she was here "to gain weight". Concordant with her stated desire to gain weight, she stated that at her lowest weight she felt she was too thin, and at the highest weight (albeit still low BMI) the patient stated that she felt attractive and believed others envied her. Revisiting the patient's presentation, desire to gain weight and positive self-evaluation at her highest weight are inconsistent with AN. A patient with AN would likely state that at her lowest weight she still felt too fat, still wanted to reduce her weight, and would be more likely to believe others perceived her body negatively as well. Furthermore, restrictive behavior regarding amounts and types of food observed over the hospital course were not motivated by desire for thinness or weight reduction but rather by a feeling of discomfort common in MELAS. Finally, no binge eating, purging, or inappropriate compensatory behaviors were present during the hospital course or on history.

In retrospect, the patient's eating symptoms were likely a conditioned behavioral response to gastrointestinal discomfort that became associated with eating. Gastrointestinal dysmotility symptoms in MELAS include esophageal distension, slowed gastric emptying and constipation, pseudoobstruction, abdominal distension, bloating, and early satiety. ${ }^{15}$ Patients may also have recurrent diarrhea, vomiting, pancreatitis, and hepatopathy due to smooth muscle dysfunction. ${ }^{8}, 15$ These symptoms may lead to avoidance of food in MELAS patients due to classical conditioning. ${ }^{15}$ Food avoidance and inadequate oral intake may lead to malnutrition and electrolyte depletion requiring medical intervention in both MELAS and $A N{ }^{8}$ Even among MELAS patients without food avoidance and eating aversion, malnutrition can result from malabsorption secondary to dysmotil- ity. Vomiting in the absence of other neurological changes has been described as a familial occurrence ${ }^{8}$ and may be mistaken for purging. ${ }^{11,16}$ Gastroscopy and colonoscopy findings are often nonspecific. ${ }^{16}$ Cellular and molecular diagnostic studies of affected organs may be needed to demonstrate mitochondrial aberrations that provide an etiology for functional impairment. ${ }^{16}$ The patient's poor dentition was considered at the time of presentation to be consistent with a history of repeatedly induced vomiting for weight control. In retrospect, however, poor dentition may have resulted from dual mechanisms, both related to MELAS. First, the patient had endorsed a history of recurrent vomiting, which is a known gastrointestinal tract manifestation of MELAS. . $^{8,11,17}$ Secondly, paradontosis is also known to result from MELAS ${ }^{17}$ and can be severe enough to require operative intervention and/or result in loss of dentition.

The presence of multiple biopsychosocial risk factors for AN in the patient's history may have biased clinicians toward an eating disorder diagnosis. These include perfectionism and emphasis on high achievement, participation in a thinness-promoting athletic culture, close but conflicted family relationships, and a history of abuse. ${ }^{18,19}$

The MELAS syndrome has been reported to have masqueraded as other psychiatric conditions. For example, hallucinations, anxiety, and hyperarousal have been reported. ${ }^{9,17,21}$ Additionally, aggressive and suicidal behavior, as well as affective instability, ideas of reference, and neglect of body care have been observed. ${ }^{17}$ Other psychiatric symptoms that have been reported among mitochondrial cytopathy patients include personality change, depression, mania, and attention deficit symptoms. ${ }^{17}$ A growing body of literature suggests that more and more patients with mitochondrial cytopathies may present with psychiatric symptoms. Greater awareness by psychiatrists of the possibility of this disorder would lead to more timely and accurate diagnosis. Patients with mitochondrial disease are diagnosed an average of $7.5 \pm 11$ years after onset of psychiatric symptoms. ${ }^{17}$ Mitochondrial cytopathy patients with psychiatric symptoms report poorer perception of their overall health, significantly lower physical functioning, and more role impairment than those without psychiatric symptoms. ${ }^{17}$

\section{CONCLUSION}

While one case of a comorbidly existing eating disorder and mitochondrial cytopathy has been described, ${ }^{19}$ never to our knowledge has the MELAS syndrome masqueraded as AN. We set out to discuss the similarities and differences among the two disorders in an attempt to better understand what may have contributed to delayed diagnosis and to heighten awareness among physicians of various specialties to the possibility of this rare mitochondrial cytopathy. 
In retrospect, our patient's symptoms are quite consistent with the clinical picture of MELAS. In assessing factors that may have contributed to the difficulties in diagnosis, at least several can be considered. First, the presentation of MELAS and the infrequency with which many well-trained physicians, especially psychiatrists, encounter the rare condition. Second, AN and MELAS bear clinical similarities. Third, the patient's psychosocial history may have made objective assessment of her symptoms less likely. In conclusion, the motivation for symptoms in patients with abnormal eating patterns should be carefully evaluated for relevance to body size, image, and weight concerns. In markedly underweight patients without these concerns, the diagnosis of an eating disorder should be reconsidered. Furthermore, presence of symptoms that cannot be explained by an eating disorder diagnosis should prompt further broadening of the differential which should include mitochondrial cytopathies such as MELAS.

\section{Sincerely, \\ Aileen Kim, MD \\ Jeremy Francis, MD \\ Gail H. Manos, MD}

\section{REFERENCES}

1. Hirano M. MELAS. Emedicine [online database]. Omaha, NE: WebMD. Accessed October 14, 2005.

2. Hirano $M$, Ricci $E$, Koenigsberger MR, et al. MELAS: An original case and clinical criteria for diagnosis. Neuromuscul Disord. 1992;2:125-135.
3. Vu TH, Hirano M, DiMauro S. Mitochondrial diseases. Neurol Clin. 2002;20:809-839.

4. lizuka T, Sakai F. Pathogenesis of stoke-like episodes in MELAS: analysis of neurovascular cellular mechanisms. Curr Neurovasc Res. 2005:2:29-45

5. Scaglia F. MELAS Syndrome. Emedicine [online database]. Omaha, NE: WebMD Accessed 0ct 14, 2005.

6. Chinnery PF, Howell N, Andrews RM, Turnbull D. Clinical Mitochondrial Genetics. $J$ Med Genet. 1999;36:425-436.

7. Dougherty FE, Ernst SG, Aprille JR. Familial recurrence of atypical symptoms in an extended pedigree with the syndrome of mitochondrial encephalomyopathy, lactic acidosis, and stroke-like episodes (MELAS). J Pediatr.1994:125:758-761.

8. Shimotake T, Furukawa T, Inoue K, Iwai N, Takeuchi Y. Familial occurrence of intestinal obstruction in children with the syndrome of mitochondrial encephalomyopathy, lactic acidosis, and stroke-like episodes (MELAS). J Pediatr Surg.1998:33:1837-1839

9. Kiejna A, DiMauro S, Adamowski T, Rymaszewska J, Leszak J. Pachalska M. Psychiatric symptoms in a patient with the clinical features of MELAS. Med Sci Monit. 2002;8:CS66-72.

10. Berbel-Garcia A, Barbera-Farre JR, Etessam JP, et al. Coenzyme 010 improves lactic acidosis, strokelike episodes, and epilepsy in a patient with MELAS imitochondrial myopathy, encephalopathy, lactic acidosis, and strokelike episodes). Clin Neuropharmacol. 2004:27:187-91.

11. Finsterer J. Overview on visceral manifestations of mitochondrial disorders. Neth $J$ Med 2006;64: 61-71.

12. Diagnostic and Statistical Manual of Mental Disorders. 4th ed, text rev. Washington, DC: American Psychiatric Association; 2000.

13. Dolan RJ, Mitchell J, Wakeling A. Structural brain changes in patients with anorexia nervosa. Psychol Med. 1998;18:349-353.

14. Braun $\mathrm{CM}$, Chouinard $\mathrm{MJ}$. Is anorexia nervosa a neuropsychological disease? Neuropsychol Rev. 1992:3:171-212.

15. Muehlenberg K, Fiedler A, Schaumann I, Muller-Felber W, Wiedmann KH. Intestina pseudoobstructions and gastric necrosis in mitochondrial myopathy. Dtsch Med Wochensch. 2002:127:611-615.

16. Chinnery PF, Turnbull DM. Vomiting, anorexia, and mitochondrial DNA disease. Lancet. 1998:351:448.

17. Fattal O, Link J, Quinn K, Cohen BH, Franco K. Psychiatric comorbidity in 36 adults with mitochondrial cytopathies. CNS Spectr. 2007:12:429-438

18. Manos G, Carlton J, dela Cruz G, Kelley WA. Eating Disorders. In: Lenhart M, Lounsbery D. North R, eds. Recruit Medicine. Washington, DC: Borden Institute; 2006:361-262.

19. Deutsch S, Rosse R, Yaseen M, Schulman H, Abraham S. Mitochondrial Myopathy complicated by eating disorder: A case report highlighting the potential interaction of genetic, metabolic, and psychodynamic factors. CNS Spectr. 2007;12:289-292.

Dr. Kim is staff psychiatrist at the National Naval Medical Center in Washington, DC. Dr. Francis is third marine regiment psychiatrist at the Marine Corps Base in Hawaii. Dr. Manos is residency training director in the Department of Psychiatry at Naval Medical Center in Portsmouth, VA. Faculty Disclosures: The authors do not have an affiliation with or financial interest in any organization that might pose a conflict of interest. Acknowledgments: The authors wish to thank Dr. Robert Wagner CAPT MC USN from the Naval Medical Center Portsmouth for his neurological expertise and contribution to the diagnosis and care of this patient.

Submitted for publication: July 23, 2008; Accepted for publication: August 14, 2009.

Please direct all correspondence to: Dr. Gail H. Manos, Department of Psychiatry, Naval Medical Center, Portsmouth, Portsmouth, VA, 23708; Tel: 757-953-5760, Fax: 757-953-5272; E-mail: ghmanos@mar.med.navy.mil. 


\section{PSYCHIATRISTS, \\ YOUR PROFESSIONAL LIABILITY INSURER MAY NO LONGER BE SAFE OR SOUND.}

\section{MAKE SURE YOUR PROFESSIONAL}

LIABILITY INSURANCE

IS PROVIDED BY A COMPANY

WITH AN"EXCELLENT" RATING.

AND DON'T SETTLE FOR

YOUR FUTURE

ANYTHING LESS.

Darwin National Assurance Company

darwin further upgrade in its rating very soon not a downgrade.

Darwin is rated "Excellent" by A.M. Best Co. and anticipates a

\section{Discounts}

- $50 \%$ for Part-time

- $10 \%$ Claims free

- $50 \%$ as a New Graduate

(Please call 1-800-421-6694 for complete details on these discounts.)

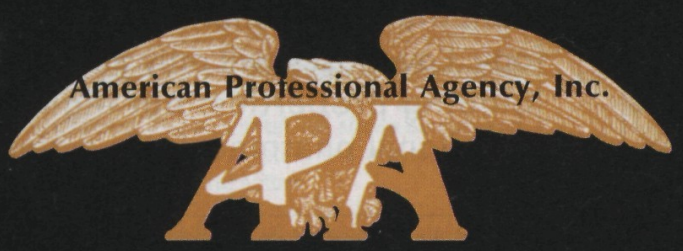

POLICY ADMINISTRATOR
Endorsed By:

AMERICAN ACADEMY OF CHILD $\mathcal{G} A D O L E S C E N T$ PSYCHIATRY

95 Broadway, Amityville, NY 11701

$$
\text { 1-800-421-6694 }
$$

w w w.americanprofessional.com 